\title{
Hyperbaric Oxygen Ameliorates Bleomycin-Induced Pulmonary Fibrosis in Mice
}

\author{
Yuan Yuan ${ }^{1 \dagger}$, Yali $\mathrm{Li}^{1+}$, Guoqiang Qiao', Yilu Zhou ${ }^{2,3}$, Zijian $\mathrm{Xu}^{2}$, Charlotte Hill ${ }^{2}$, \\ Zhenglin Jiang ${ }^{1 *}$ and Yihua Wang ${ }^{2,3 *}$
}

${ }^{1}$ Department of Neurophysiology and Neuropharmacology, Institute of Special Environmental Medicine and Co-Innovation Center of Neuroregeneration, Nantong University, Nantong, China, ${ }^{2}$ Biological Sciences, Faculty of Environmental and Life Sciences, University of Southampton, Southampton, United Kingdom, ${ }^{3}$ Institute for Life Sciences, University of Southampton,

Southampton, United Kingdom

\section{OPEN ACCESS}

Edited by:

Honggang Zhou,

Nankai University, China

Reviewed by:

Snehal M. Gaikwad,

National Cancer Institute,

United States

Qiangmin Xie,

Zhejiang University, China

Weigang $\mathrm{Xu}$,

The Second Military Medical

University, China

*Correspondence:

Yihua Wang

yihua.wang@soton.ac.uk

Zhenglin Jiang

jiangzl@ntu.edu.cn

tThese authors have contributed equally to this work and share first authorship

Specialty section: This article was submitted to Molecular Diagnostics and

Therapeutics,

a section of the journal

Frontiers in Molecular Biosciences

Received: 03 March 2021 Accepted: 30 April 2021

Published: 04 June 2021

Citation:

Yuan Y, Li Y, Qiao G, Zhou Y, Xu Z, Hill $C$, Jiang $Z$ and Wang $Y$ (2021)

Hyperbaric Oxygen Ameliorates

Bleomycin-Induced Pulmonary

Fibrosis in Mice.

Front. Mol. Biosci. 8:675437.

doi: 10.3389/fmolb.2021.675437
The prevalence of pulmonary fibrosis is increasing with an aging population and its burden is likely to increase following COVID-19, with large financial and medical implications. As approved therapies in pulmonary fibrosis only slow disease progression, there is a significant unmet medical need. Hyperbaric oxygen $(\mathrm{HBO})$ is the inhaling of pure oxygen, under the pressure of greater than one atmosphere absolute, and it has been reported to improve pulmonary function in patients with pulmonary fibrosis. Our recent study suggested that repetitive HBO exposure may affect biological processes in mice lungs such as response to wounding and extracellular matrix. To extend these findings, a bleomycin-induced pulmonary fibrosis mouse model was used to evaluate the effect of repetitive $\mathrm{HBO}$ exposure on pulmonary fibrosis. Building on our previous findings, we provide evidence that $\mathrm{HBO}$ exposure attenuates bleomycin-induced pulmonary fibrosis in mice. In vitro, $\mathrm{HBO}$ exposure could reverse, at least partially, transforming growth factor (TGF)- $\beta$-induced fibroblast activation, and this effect may be mediated by downregulating TGF- $\beta$-induced expression of hypoxia inducible factor (HIF)- $1 \alpha$. These findings support $\mathrm{HBO}$ as a potentially life-changing therapy for patients with pulmonary fibrosis, although further research is needed to fully evaluate this.

Keywords: pulmonary fibrosis, hyperbaric oxygen, fibroblast, differentiation, extracellular matrix, HIF-1a

\section{INTRODUCTION}

Pulmonary fibrosis, an interstitial lung disease, is characterized by enhanced deposition and remodeling of the extracellular matrix (ECM), leading to disrupted gas exchange, and ultimately respiratory failure and death (Richeldi et al., 2017). The prevalence of pulmonary fibrosis is increasing with an aging population (Richeldi et al., 2017) and its burden after COVID-19 recovery could be substantial (George et al., 2020). Idiopathic pulmonary fibrosis (IPF), the most common type of progressive fibrotic interstitial lung disease, affects five million people worldwide (Meltzer and Noble, 2008), with a median survival of 3 years (Goodwin and Jenkins, 2016; Richeldi et al., 2017). The current approved therapies for pulmonary fibrosis only slow the disease progression, and as such there is a demand for new treatment options.

Current clinical management of IPF patients includes anti-fibrotic drugs and nonpharmacological support (Richeldi et al., 2017). For patients with advanced disease, reducing symptoms and improving quality of life are required (Zou et al., 2020). Long-term oxygen therapy, with high 
flow and high concentration of oxygen, is often used to decrease dyspnea and improve exercise tolerance (Koyauchi et al., 2018; Faverio et al., 2019). It is also reported that oxygen supplementation increased exercise capacity for patients with interstitial lung diseases including IPF (Bell et al., 2017; Dowman et al., 2017). Moreover, the benefit of high flow oxygen compared to placebo air was found to improve the quality of life for patients with fibrotic lung disease in a clinical trial (Visca et al., 2018).

Hyperbaric oxygen involves inhaling pure oxygen in a closed chamber pressurized to greater than one atmosphere absolute (ATA). The clinical applications of $\mathrm{HBO}$ in ischemic and nonhealing wounds have been reported since the mid-20th century (Lam et al., 2017). An updated list of its applications can be found on the Undersea and Hyperbaric Medical Society Web site (https://www.uhms.org/resources/hbo-indications. html) and have also been reviewed elsewhere (Choudhury, 2018; Kirby, 2019). Interestingly, HBO therapy has been reported to improve pulmonary function in IPF patients (Ma and Du, 2003; Qiu et al., 2013). In another report, HBO exposure reduced radiation-induced side effects including fibrosis in a rat bladder irradiation model (Oscarsson et al., 2017). Mechanistically, our recent study suggested that repetitive $\mathrm{HBO}$ exposure may affect biological processes in mice lungs such as response to wounding and ECM (Yuan et al., 2020). To extend these findings, a bleomycin-induced pulmonary fibrosis mouse model was used to evaluate the effect of repetitive $\mathrm{HBO}$ exposure on pulmonary fibrosis. Building on our previous report (Yuan et al., 2020), we provide evidence that HBO exposure attenuates bleomycin-induced pulmonary fibrosis in mice. In vitro, $\mathrm{HBO}$ exposure could reverse, at least partially, transforming growth factor (TGF)- $\beta$-induced fibroblast activation. These findings support $\mathrm{HBO}$ as a potentially lifechanging therapy for patients with pulmonary fibrosis, although further research is needed to fully evaluate this.

\section{MATERIALS AND METHODS}

\section{Pathway Enrichment Analysis}

The RNA-seq data analyzed were based on our previous study (Yuan et al., 2020) (GSE143348). Briefly, lungs were collected from control mice or $\mathrm{HBO}$-treated mice that were repetitively exposed to $2.5 \mathrm{ATA} \mathrm{HBO}, 90 \mathrm{~min} /$ time, once a day for 11 consecutive days. Control mice were placed in the chamber for the same duration without pure oxygen pressurization. Lung samples were collected on the next day of the last HBO exposure. Total RNA was isolated for library construction, and it was sequenced with the paired-end strategy $(2 \times 150)$ on the Illumina NovaSeq 6000 platform following the standard protocols. Enrichment analyses of down-regulated differentially expressed genes (DEGs) were generated by Metascape with default parameters (https:// metascape.org/gp/index.html\#/main/step1). All significantly enriched Gene Ontology (GO) terms and their $p$ values were imported into REVIGO (http://revigo.irb.hr/) to remove redundant GO terms. GO: 0062023 (collagen containing extracellular matrix) and GO: 0031012 (extracellular matrix) gene lists were downloaded from MSigDB Collections (http://www.gsea-msigdb.org/gsea/msigdb/) and converted into corresponding mouse genes. Based on these gene lists, the pathway enrichment score for each sample was calculated by using gene set variation analysis in the GSVA (v1.36.2) package (Hanzelmann et al., 2013).

\section{Bleomycin-Induced Pulmonary Fibrosis in Mice}

Six- to eight-week-old male C57BL/6 mice were purchased from the Experimental Animal Center of Nantong University (institutional license: SYXK(SU)-2012-0030). Mice were maintained under a $12 \mathrm{~h}$ light $/ 12 \mathrm{~h}$ dark cycle, and normal diet and water were provided ad libitum throughout the study. Animal experiments were approved by the Animal Ethics Committee at Nantong University (approval number: 20140901-001).

One dose of $2.0 \mathrm{U} / \mathrm{kg}$ of bleomycin (Hisun Pfizer Pharmaceutical Co., Ltd., Zhejiang, China) was intratracheally instilled to induce pulmonary fibrosis in mice. After bleomycin administration, body weights were monitored every third day. According to the previous report, weight loss is an indicator of successful model construction (Vandivort et al., 2016). Mice with a weight loss of less than $5 \%$ at day 7 or less than $10 \%$ at day 10 post the bleomycin challenge were excluded from further study.

\section{Hyperbaric Oxygen Exposure of Mice or Cells}

A hyperbaric chamber designed for small animal research was used for HBO exposure, as described previously (Yuan et al., 2020). Briefly, after the chamber was flushed with pure oxygen for $5 \mathrm{~min}$, the pressure ramped up to 2.5 ATA (1.5 atm) by inflating $100 \%$ oxygen slowly in $5 \mathrm{~min}$, then sustained at 2.5 ATA for $90 \mathrm{~min}$, and finally decompressed slowly in $5 \mathrm{~min}$. The concentrations of carbon dioxide and oxygen were monitored by SDA carbon dioxide and oxygen monitors (Analox, North Yorkshire, England) during the exposure. Bleomycin-challenged mice were randomized into control or HBO-treated group, in which HBO exposure was applied daily from day 7 after intratracheal bleomycin instillation until day 20, and samples were collected at day 21. Mice in the control group were maintained in the normoxia condition throughout the study. Before sample collections, mice were anesthetized with composited anesthetics (257 mM chloral hydrate, $176 \mathrm{mM}$ magnesium sulfate, $36 \mathrm{mM}$ pentobarbital sodium, $14.25 \%$ ethanol, and $33.8 \%$ propylene glycol).

To treat cells with $\mathrm{HBO}$, a hyperbaric chamber designed for cell culture was used. An embedded circulating water device was used to keep the environmental temperature at $37^{\circ} \mathrm{C}$. $\mathrm{HBO}$ exposure was applied at 2.5 ATA for $90 \mathrm{~min}$. To maintain the $\mathrm{pH}$ of the cell culture medium, the mixed gas with $98 \%$ oxygen and $2 \%$ carbon dioxide was used to maintain the partial pressure of carbon dioxide at $5 \mathrm{kPa}$ under 2.5 ATA pressure. 


\section{Hematoxylin and Eosin and Masson's Trichrome Staining}

The left lung lobes of the mice were used for morphological examinations. Lungs were fixed with $4 \%$ paraformaldehyde for $24 \mathrm{~h}$, dehydrated by gradient ethanol, embedded in paraffin, and sliced $5 \mu \mathrm{m}$ thick successively. For staining experiments, the tissue sections were dewaxed and rehydrated. For $\mathrm{H} / \mathrm{E}$ staining, a H/E stain kit (Beyotime Biotechnology, Shanghai, China) was used according to the protocol. For Masson's trichrome stain, a Masson stain kit (Nanjing Jiancheng Bioengineering Institute, Jiangsu, China) was used following the manufacturer's instructions. The DM4000B microscope (Leica, Wetzlar, Germany) was used for imaging.

\section{Ashcroft Score Evaluation}

Ashcroft scores were evaluated as previously described (Hubner et al., 2008). To ensure the accuracy of the results, a double-blind strategy was adopted when scoring. Two researchers were asked to score without knowing group information, and the means of the scores for each sample were used for further statistical analysis.

\section{Hydroxyproline Quantification}

Lung tissues were harvested from mice at day 21 after bleomycin administration. Following excision, tissues were immediately flash-frozen in liquid nitrogen. A hydroxyproline assay kit from KeyGEN BioTECH (Jiangsu, China) was used to detected hydroxyproline levels in lungs following the manufacturer's instructions. Hydroxyproline contents were normalized to the lung tissue mass.

\section{Cell Culture and Reagents}

Human lung fibroblast HFL1 cells were purchased from the Institute of Cell Research (Chinese Academy of Sciences, China) and were cultured in the Nutrient Mixture F-12 Ham (Sigma-Aldrich, MA, United States) cell culture medium containing $10 \%$ fetal bovine serum (Gibco, NY, United States) and $1 \%$ penicillin/streptomycin. Cells were cultured in a $37^{\circ} \mathrm{C}$ incubator containing $5 \% \mathrm{CO}_{2}$. No mycoplasma contamination was detected in the cell line used. TGF- $\beta$ was from PeproTech (NJ, United States).

\section{Western Blot Analysis}

Protein samples from cells or lung tissues were lysed with RIPA buffer (Beyotime Biotechnology, Shanghai, China) containing the protease inhibitor (Meilunbio, Liaoning, China). Primary antibodies were from Cell Signaling Technology ( $\alpha$-SMA, 14968), Sigma-Aldrich ( $\beta$-actin, A5316), and R\&D Systems (HIF-1a, AF 1935). Signals were detected using an ECL detection system with a Tanon 5200 Multi imaging system (Shanghai, China) and evaluated by ImageJ $1.42 \mathrm{q}$ software (National Institutes of Health).

\section{Real-Time qPCR Analysis}

Total RNA samples were isolated from cultured cells or lung tissues with the TRIzol reagent (Invitrogen, CA, United States), following the manufacturer's instructions and quantified using a One Drop OD-1000+ Spectrophotometer (One Drop, Shanghai, China). HiScript II RT SuperMix for qPCR was used for reverse transcriptions (+gDNA wiper) (Vazyme, Jiangsu, China). Universal SYBR qPCR Master Mix was used for qPCR assays (Vazyme, Jiangsu, China). Relative transcript levels of target genes were normalized to $\beta$-actin ( $A C T B$ in human and $A c t b$ in mouse). Primers for the genes detected were as follows:

Human ACTA2-Forward: ACTGCCTTGGTGTGTGACAA, Human ACTA2-Reverse: CACCATCACCCCCTGATGTC; Human FN1-Forward: AGGAAGCCGAGGTTTTAACTG, Human FN1-Reverse: AGGACGCTCATAAGTGTCACC; Human COL1A1-Forward: GAGGGCCAAGACGAAGACATC, Human COL1A1-Reverse: CAGATCACGTCATCGCACAAC; Human ACTB-Forward: GGATTCCTATGTGGGCGACGA, Human ACTB-Reverse: GCGTACAGGGATAGCACAGC; Mouse Acta2-Forward: TCCCTGGAGAAGAGCTACGAAC, Mouse Acta2-Reverse: AGGACGTTGTTAGCATAGAGATCC; Mouse Col1a1-Forward: AGCACGTCTGGTTTGGAGAG, Mouse Col1a1-Reverse: GACATTAGGCGCAGGAAGGT; Mouse Fn1-Forward: CCCCAACTGGTTACCCTTCC, Mouse Fn1-Reverse: TGTCCGCCTAAAGCCATGTT; Mouse $A c t b$-Forward: ACACCCGCCACCAGTTC, Mouse $A c t b$-Reverse: TACAGCCCGGGGAGCAT.

\section{Statistical Analysis and Repeatability of Experiments}

Each experiment was repeated at least twice. Data are presented as mean and standard deviation (s.d.). A two tailed, unpaired, parametric, or nonparametric t-test was used to compare two groups of values, depending on whether the data distribution passed the normality test. One outlier in Ashcroft scores identified by ROUT analysis $(Q=1 \%)$ was removed from statistical analysis. One-way ANOVA (single-factor analysis of variance) was used to compare more than two groups of data. Two-way ANOVA (two-factors analysis of variance) was applied to analyze the difference of the body weight change curve. GraphPad Prism 8.0 software was used for analysis and $p<$ 0.05 was considered as statistically significant.

\section{RESULTS}

\section{Repetitive Hyperbaric Oxygen Treatments Downregulates Extracellular Matrix Gene Expression in Mouse Lungs}

Our previous study suggested that repetitive HBO treaments may affect biological processes in the lungs, such as response to wounding and extracellular matrix (Yuan et al., 2020). We reported that in the down-regulated genes in mice lungs following repetitive $\mathrm{HBO}$ exposure (GSE143348), enriched terms for cellular component classification including the "collagen containing extracellular matrix" and "extracellular 


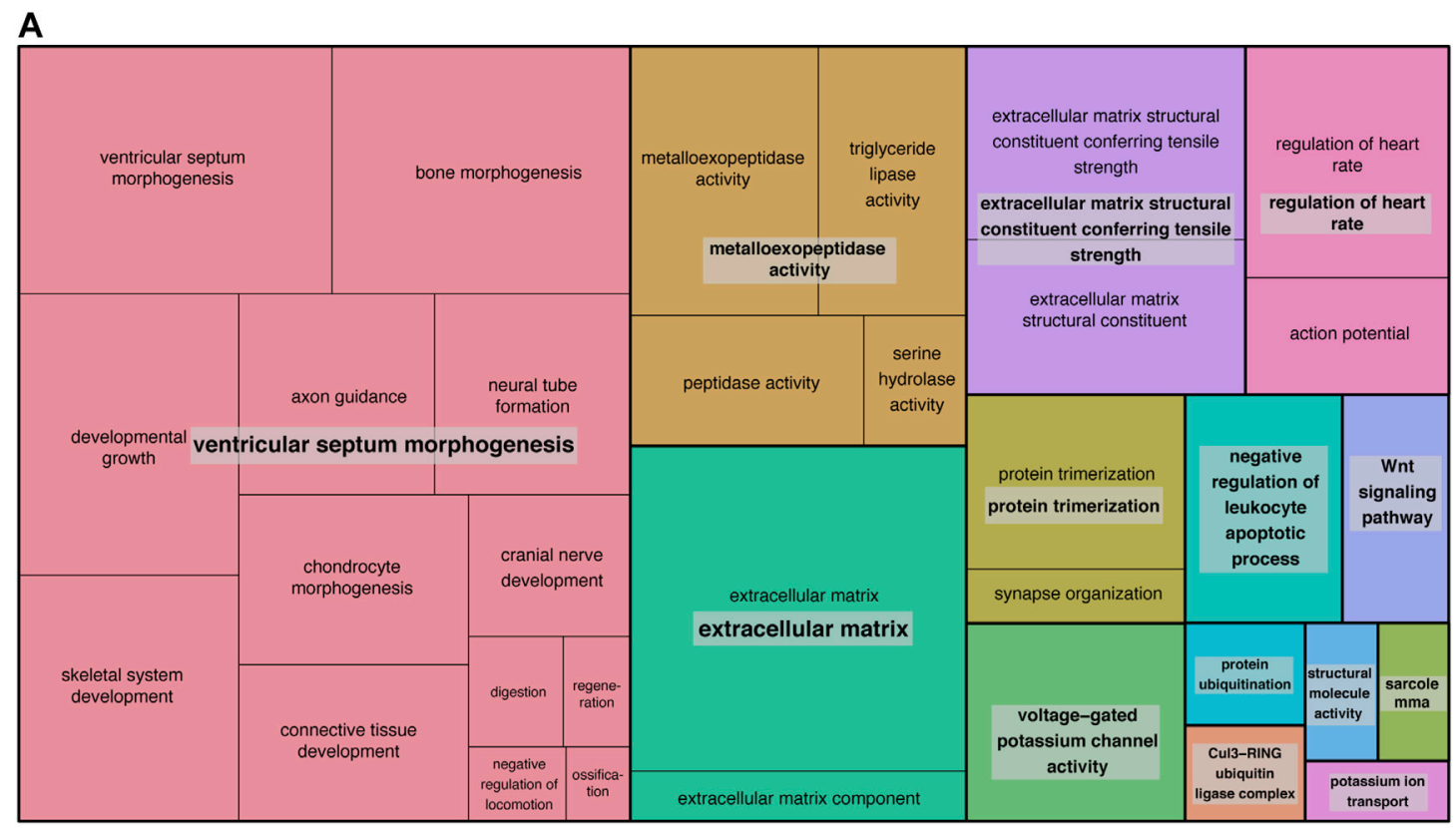

B

\section{Collagen containing}

(GO: 0062023)

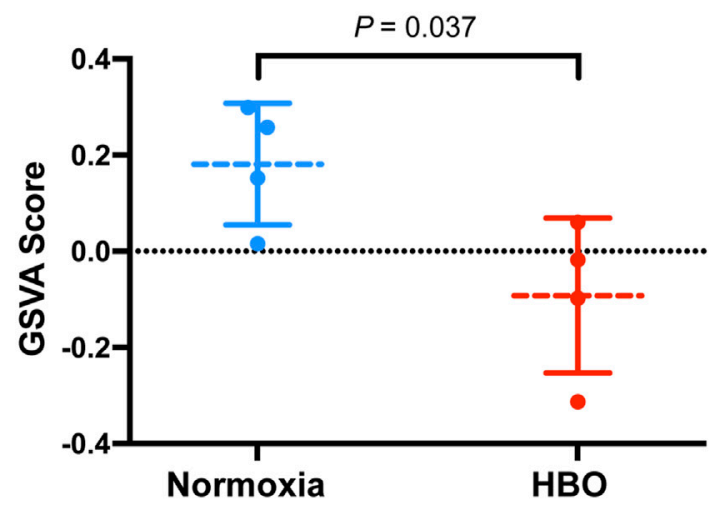

C

Extracellular matrix

(GO: 0031012)

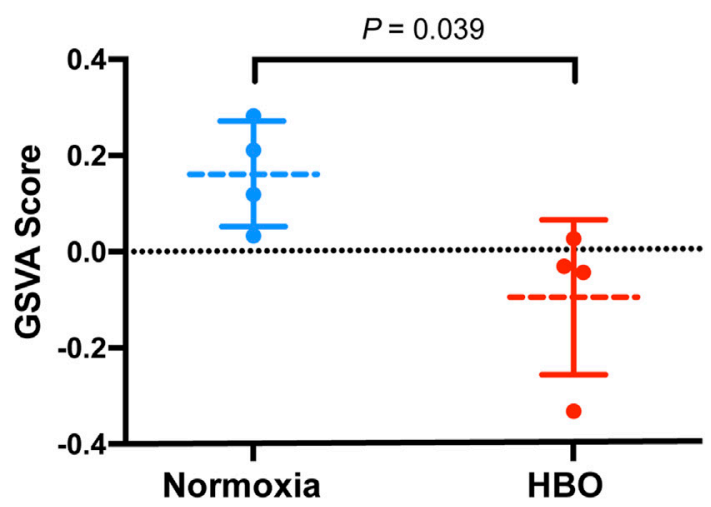

FIGURE 1 | Repetitive HBO treatments downregulate extracellular matrix gene expression in mouse lungs. (A) REVIGO TreeMap showing Gene Ontology (GO) analysis of downregulated differentially expressed genes (DEGs) in mice lungs exposed to repetitive HBO (GSE143348). Common colors represent groupings based on parent $\mathrm{GO}$ terms, and each rectangle is proportional to the relative enrichment of the GO term compared to the whole genome. Genes with a false discovery rate $($ FDR) < 0.05 were considered as DEGs. (B,C) Graphs showing GSVA scores calculated based on gene lists from GO: 0062023 (collagen containing the extracellular matrix) (B) or GO:0031012 (extracellular matrix) (C) in HBO-treated and control lungs. Data were analyzed with the unpaired $t$-test. Data are mean \pm s.d., with $p$ values indicated. $n=4$ samples per group.

matrix component," suggesting that the extracellular matrix may be affected (Yuan et al., 2020). These findings were also reflected using the REVIGO TreeMap, which found the "extracellular matrix" as a GO-enriched term (Figure 1A).

The effect of HBO treatment on ECM genes was further demonstrated through gene set variation analysis (GSVA) using a gene list from GO: 0062023 (collagen containing extracellular matrix); GSVA scores calculated based on this gene list were significantly lower in HBO-treated vs. control (normoxia) mice lungs ( $p=0.037$; Figure 1B). Similar results were obtained using another gene list from GO: 0031012 (extracellular matrix) $(p=0.039$; Figure 1C). Together, these results demonstrate the potential impact of $\mathrm{HBO}$ treatment on ECM deposition in mice lungs. 


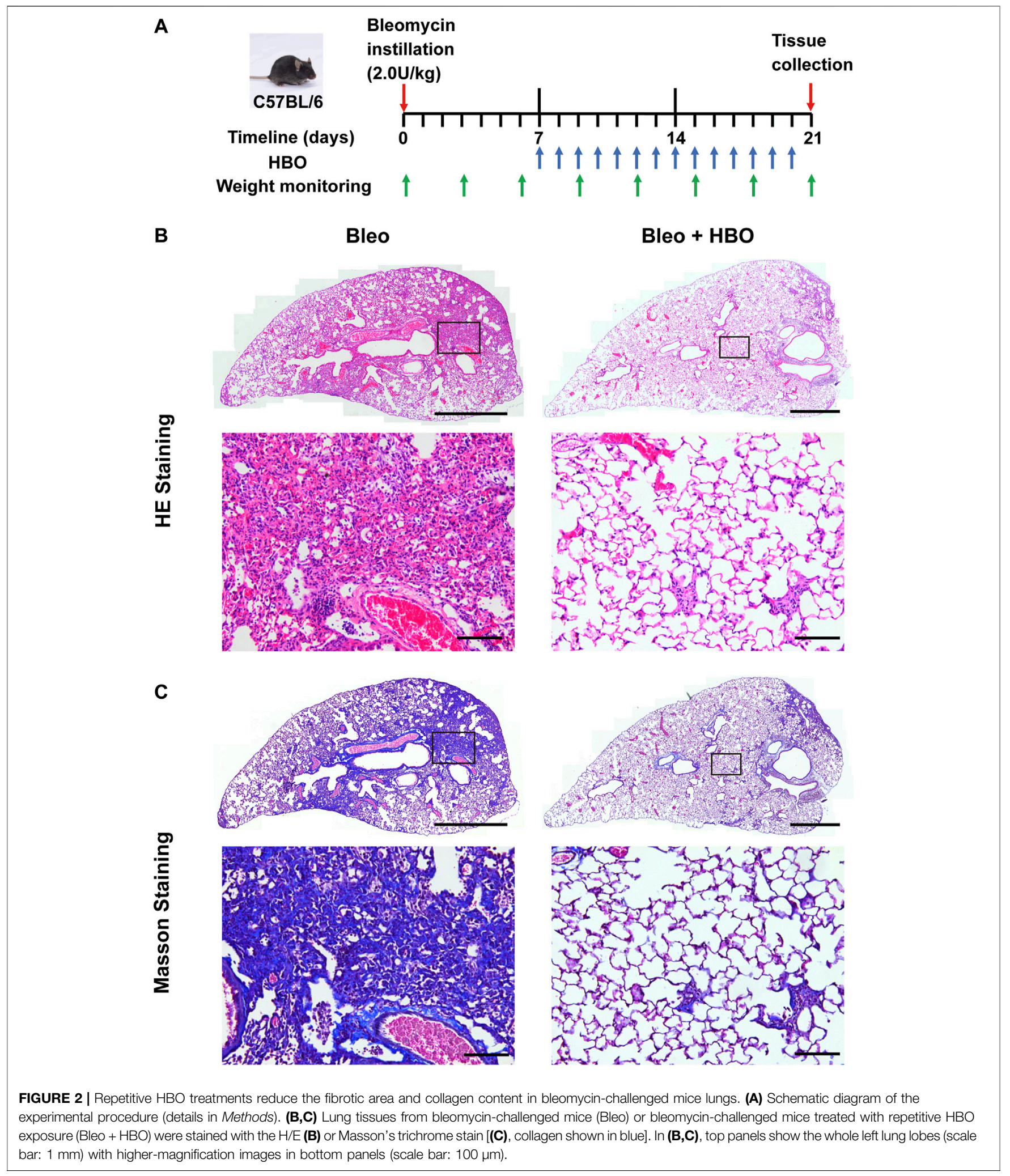




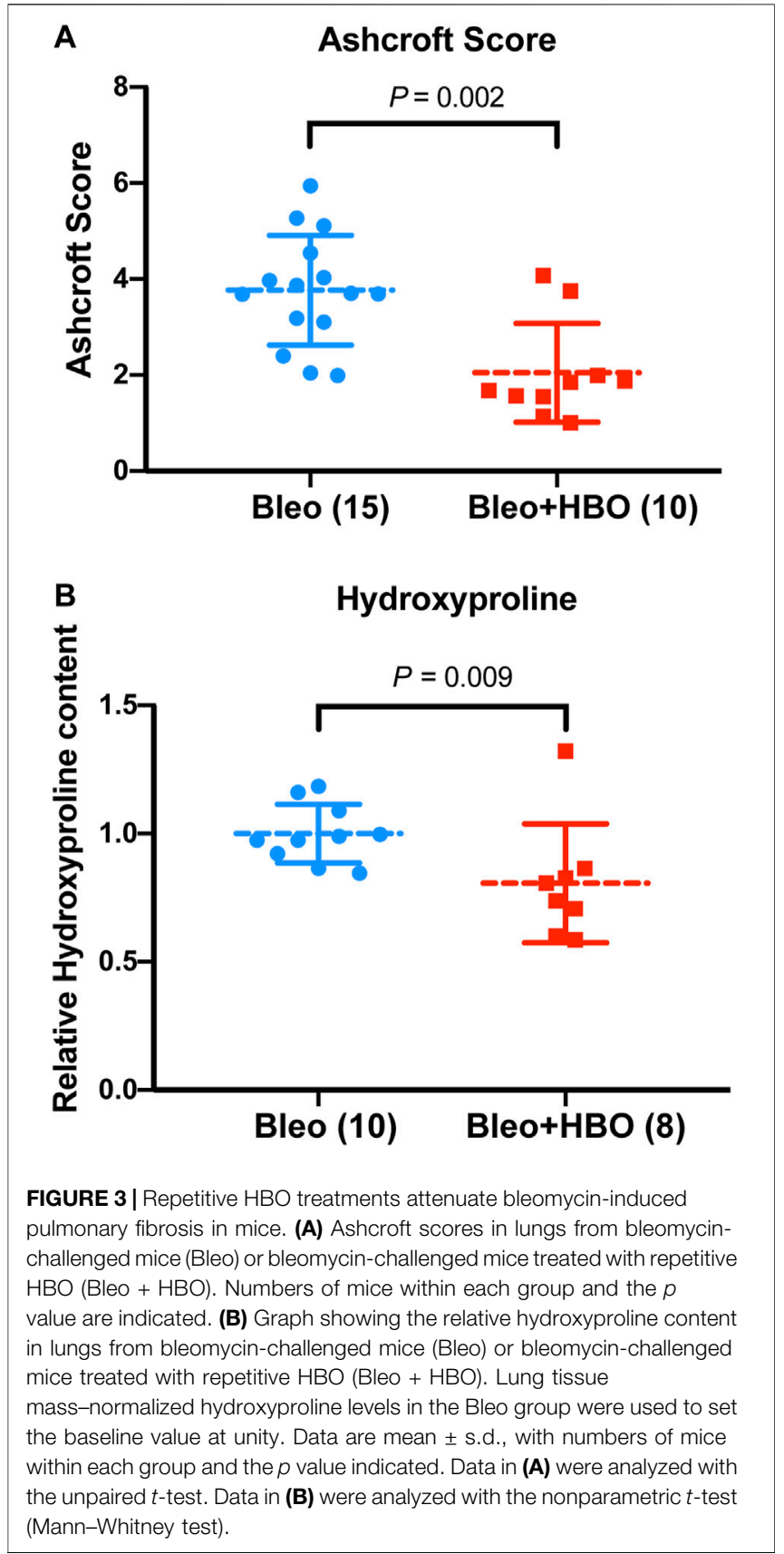

\section{Repetitive Hyperbaric Oxygen Treatments Attenuate Bleomycin-Induced Pulmonary Fibrosis in Mice}

Given the above observation, we next tested whether HBO exposure could affect the development of pulmonary fibrosis, where aberrant ECM deposition is a key feature. To test this hypothesis, bleomycin-induced pulmonary fibrosis in C57BL/6 mice was used (Supplmentary Figure S1). HBO exposure was

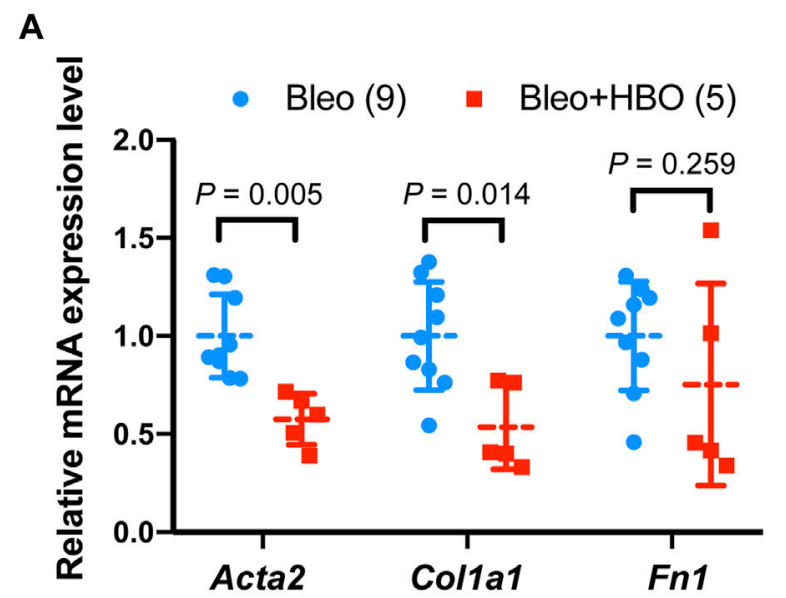

B
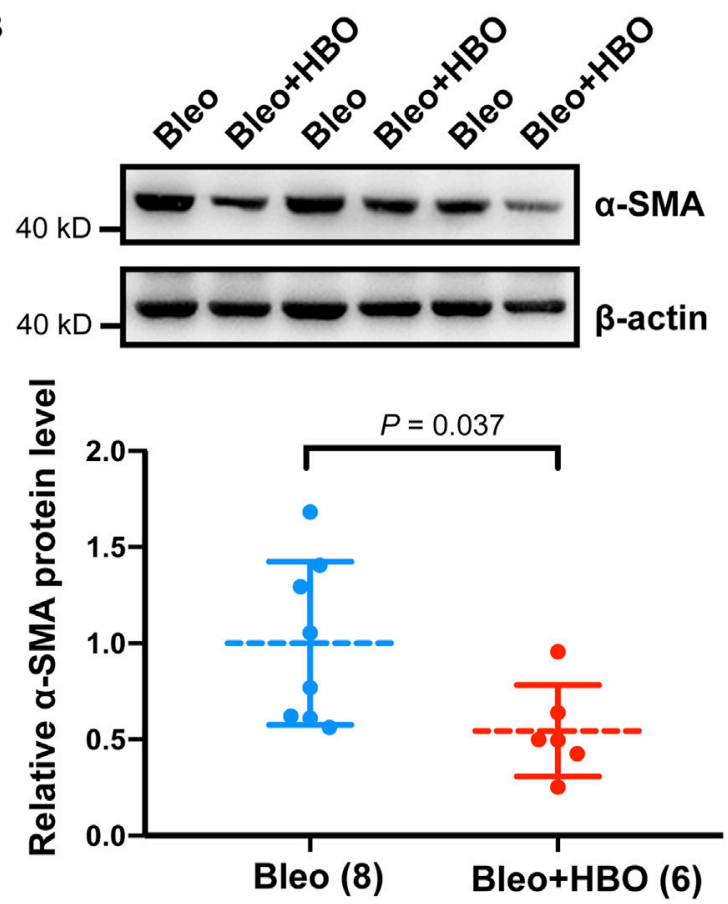

FIGURE 4 | Effects of repetitive HBO treatments on fibroblast activation and ECM deposition in mice lungs. (A) Fold change in the mRNA levels of Acta2 ( $\alpha$-SMA), Col1a1 (collagen I), and Fn1 (fibronectin) in the lungs from bleomycin-challenged mice (Bleo) or bleomycinchallenged mice treated with repetitive $\mathrm{HBO}(\mathrm{BleO}+\mathrm{HBO})$. Actb ( $\beta$-actin) -normalized mRNA levels in the Bleo group were used to set the baseline value at unity. Data are mean \pm s.d., with numbers of mice within each group and the $p$ value indicated. (B) Protein expression of $\alpha$-SMA in lungs from bleomycin-challenged mice (Bleo) or bleomycin-challenged mice treated with repetitive $\mathrm{HBO}(\mathrm{BleO}+\mathrm{HBO}) . \beta$-actin was used as a loading control. In the graph, $\beta$-actin-normalized protein levels in the Bleo group were used to set the baseline value at unity. Data are mean \pm s.d., with numbers of mice within each group and the $p$ value indicated. Data in (A) were analyzed with the unpaired multiple $t$-test. Data in (B) were analyzed with the unpaired $t$-test. 
A
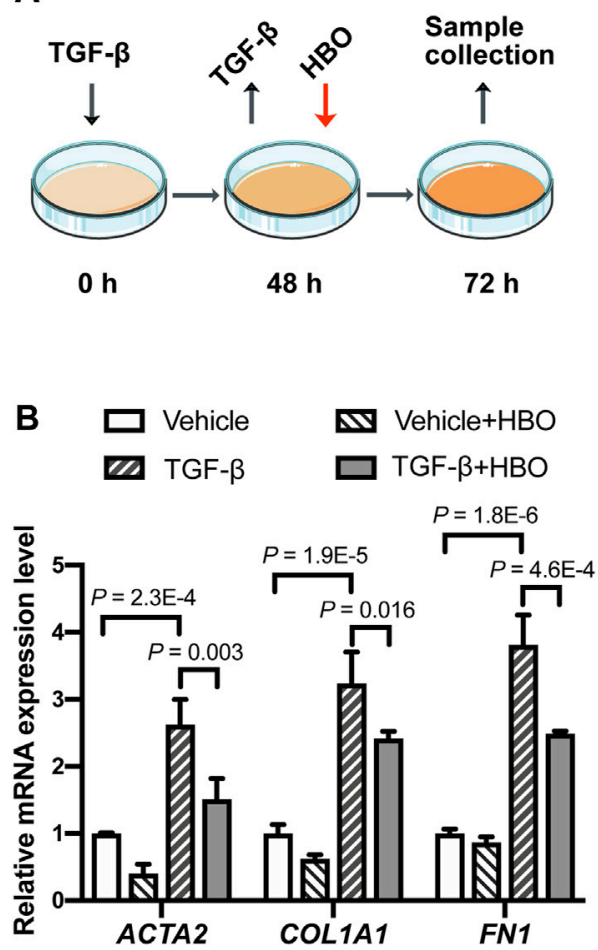
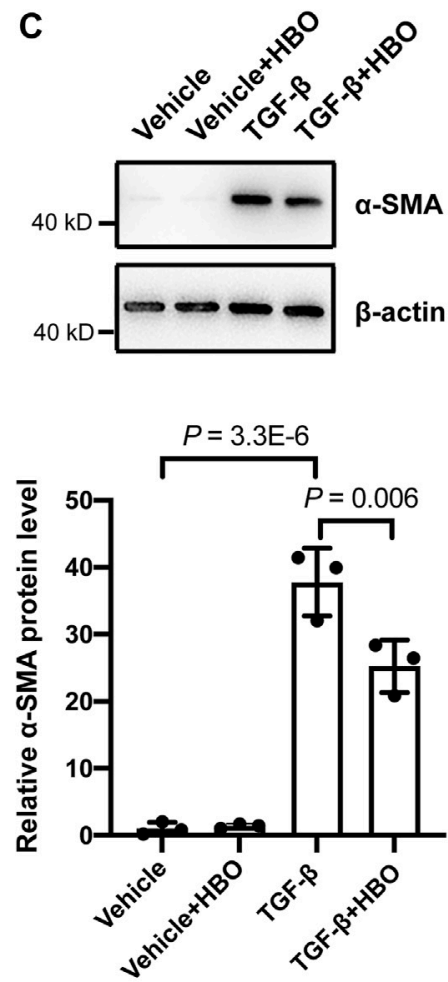

FIGURE 5 | Effects of HBO treatment on TGF- $\beta$-induced fibroblast activation in HFL1 cells. (A) Schematic diagram of the experimental procedure. In brief, TGF- $\beta$ $(5 \mathrm{ng} / \mathrm{ml})$ was added to HFL1 cells for $48 \mathrm{~h}$ to induce fibroblast activation, after which TGF- $\beta$ was removed, and cells were exposed to $2.5 \mathrm{ATA}$ HBO for 90 min immediately. Samples were collected at $72 \mathrm{~h}$ after the beginning of TGF- $\beta$ treatment. (B) The fold change in the mRNA levels of $A C T A 2$ ( $\alpha$-SMA), COL $1 A 1$ (collagen I), and FN1(fibronectin) in HFL1 cells with indicated treatments. ACTB ( $\beta$-actin)-normalized mRNA levels in control cells (vehicle) were used to set the baseline value at unity. (C) Protein expression of $\alpha$-SMA in HFL1 cells with indicated treatments. $\beta$-actin was used as a loading control. In the graph, $\beta$-actin-normalized protein levels in control cells (vehicle) were used to set the baseline value at unity. Data in $\mathbf{( B , C )}$ are mean \pm s.d., with $p$ values indicated. $n=3$ samples each group. Data were analyzed with one-way ANOVA.

applied daily from day 7 , after intratracheal bleomycin instillation until day 20, one day before sample collections (Figure 2A). Bleomycin-challenged mice showed a clear development of pulmonary fibrosis, with thickened alveoli septae and collagen deposition in the interstitium visualized in the $\mathrm{H} / \mathrm{E}$ stain (Figure 2B) and Masson's trichrome stain (Figure 2C). In contrast, fibrotic areas and collagen deposition were markedly reduced in lungs from bleomycin-challenged mice treated with repetitive $\mathrm{HBO}$ (Figures $\mathbf{2 B}, \mathbf{C}$, right panels).

To quantify the severity of fibrosis, Ashcroft scores were evaluated, and a clear reduction was observed in the lungs from bleomycin-challenged mice treated with repetitive $\mathrm{HBO}$ compared to those from bleomycin-challenged mice ( $p=$ 0.002; Figure 3A). Hydroxyproline is a major component of fibrillar collagen of all types. Consistent with the morphological changes and Ashcroft scores above, the hydroxyproline content was significantly reduced in lungs from bleomycin-challenged mice treated with repetitive HBO $(p=0.009$; Figure 3B). Effects of $\mathrm{HBO}$ on body weight in mice after the bleomycin challenge were minimal ( $p=0.820$; Supplementary Figure S2). Taken together, these data highlight an impact of repetitive $\mathrm{HBO}$ exposure on bleomycin-induced pulmonary fibrosis in mice.

\section{Effect of Repetitive Hyperbaric Oxygen Treatments on Fibroblast Activation and Extracellular Matrix Deposition in Mice Lungs}

We next checked the expression levels of Acta2 (encoding a-smooth muscle actin, a-SMA, a myofibroblast marker) and other ECM genes, including Colla1 (encoding type I collagen) and $F n 1$ (encoding fibronectin) in mice lungs. As expected, the mRNA levels of Acta2 (a-SMA), Colla1 (collagen I), and Fn1 (fibronectin) were significantly increased in the lungs from bleomycin-challenged mice compared to that of the control mice (all $p$ values were less than 0.05; Supplementary Figure S3). When the bleomycin-challenged mice were exposed to repetitive HBO treatments, the mRNA level of Acta2 ( $\alpha$-SMA) and Colla1 were both significantly reduced $(p=0.005$ and 0.014 , respectively; Figure $\mathbf{4 A}$ ). Under the same conditions, the expression of $F n 1$ was also decreased, although statistical significance was not reached ( $p=0.259$; Figure 4A). Similar results were obtained when measuring the protein level of $\alpha$-SMA using western blot ( $p=0.037$; Figure $4 B$ ). These results indicate that repetitive $\mathrm{HBO}$ exposure could potentially reduce myofibroblast differentiation and activation in vivo. 

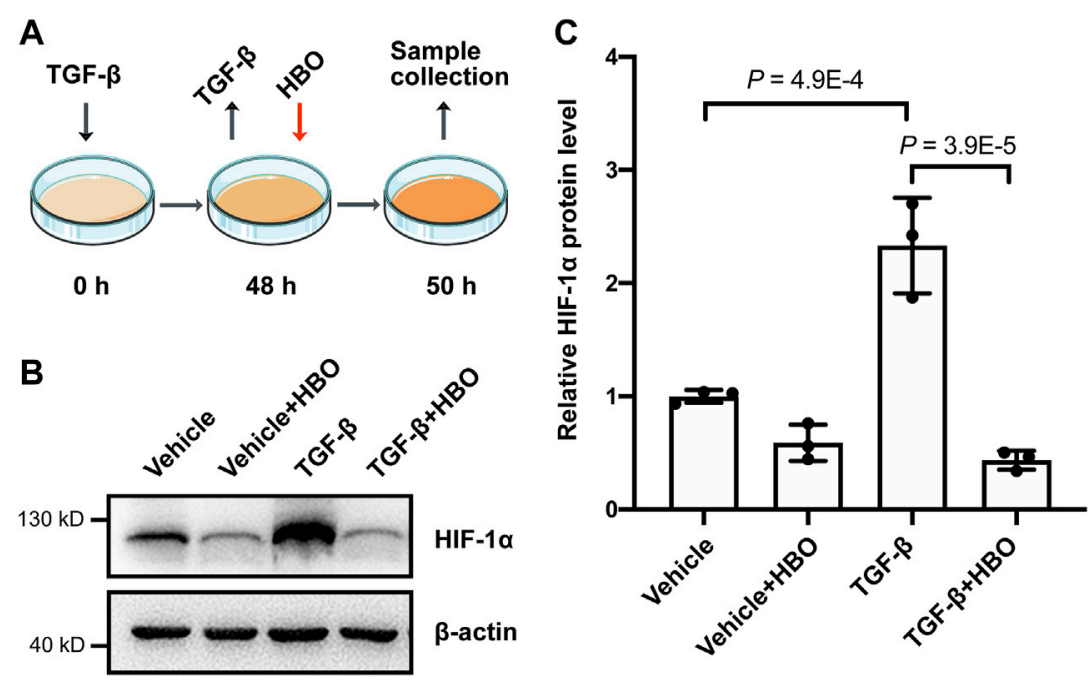

FIGURE 6 | Effects of HBO treatment on TGF- $\beta$-induced HIF-1 $\alpha$ expression in HFL1 cells. (A) Schematic diagram of the experimental procedure. In brief, TGF- $\beta$ $(5 \mathrm{ng} / \mathrm{ml}$ ) was added to HFL1 cells for $48 \mathrm{~h}$ to induce fibroblast activation, after which TGF- $\beta$ was removed, and followed 2.5 ATA HBO exposure for 90 min immediately. Samples were collected at the end of HBO exposure. (B) Protein expression of HIF-1a in HFL1 cells with indicated treatments. $\beta$-actin was used as a loading control. (C) Fold change in the protein level of HIF-1 $\alpha$ in HFL1 cells with indicated treatments. $\beta$-actin-normalized protein levels in control cells (vehicle) were used to set the baseline value at unity. Data are mean \pm s.d., with $p$ values indicated. $n=3$ samples each group. Data were analyzed with one-way ANOVA.

\section{Effect of Hyperbaric Oxygen Treatment on TGF- $\beta$-Induced Fibroblast Activation and HIF-1a Levels in Human Lung Fibroblasts}

To validate the findings in vitro, the effects of $\mathrm{HBO}$ treatment on TGF- $\beta$-induced fibroblast activation in human lung fibroblasts HFL1 were examined (Figure 5A). After incubating HFL1 cells with TGF- $\beta$ for $48 \mathrm{~h}$, the mRNA levels of ACTA2 ( $\alpha$-SMA), COL1A1 (collagen I), and FN1(fibronectin) were all induced in HFL1 cells compared to that of control cells (all $p$ values were less than 0.05; Supplementary Figure S4), indicating fibroblasts were activated. TGF- $\beta$ was then removed and $\mathrm{HBO}$ exposure was applied to HFL1 cells for $90 \mathrm{~min}$ (Figure 5A). At $72 \mathrm{~h}$ after TGF- $\beta$ treatment, ACTA2 ( $\alpha$-SMA), COL1A1, and FN1 sustained at high expression levels in TGF- $\beta$-treated groups compared to that of controls (all $p$ values were less than 0.05 ; Figure 5B). In TGF- $\beta$-treated cells, the mRNA levels of ACTA2 (a-SMA), COL1A1, and FN1 were significantly reduced when exposed to $\mathrm{HBO}$ (all $p$ values were less than 0.05; Figure 5B). In the absence of TGF- $\beta$, the mRNA levels of ACTA2 ( $\alpha$-SMA), $C O L 1 A 1$, and $F N 1$ were also decreased when exposed to HBO, although statistical significance was not reached (Figure 5B). Similar results were obtained when measuring the protein level of a-SMA using western blot (Figure 5C). In addition, to test if HBO treatment could block TGF- $\beta$-induced fibroblast differentiation, $\mathrm{HBO}$ exposure was applied immediately following TGF- $\beta$ treatment (Supplementary Figure S5A). Q-PCR showed that this treatment could also reduce TGF$\beta$-induced ACTA2 ( $\alpha$-SMA) mRNA levels in HFL1 cells ( $p=$ 0.043) (Supplementary Figure S5B). These results suggested that $\mathrm{HBO}$ exposure could reverse and block, at least partially, TGF- $\beta$-induced fibroblast activation.
Finally, we checked the effects of HBO treatment on HIF-1a levels following TGF- $\beta$ treatment in human lung fibroblasts (Figure 6A). In consistence with previous studies (Yamazaki et al., 2017; Senavirathna et al., 2020), TGF- $\beta$ treatment significantly upregulated the protein levels of HIF-1 $1 \alpha$ in HFL1 $(p=4.9 \mathrm{E}-4$; Figures 6B,C). As expected, HBO exposure dramatically reduced TGF- $\beta$-induced HIF-1 $\alpha$ protein expression ( $p=3.9 \mathrm{E}-5$; Figures $6 \mathrm{~B}, \mathrm{C})$. In addition, we were able to show that $\mathrm{HBO}$ exposure can also block TGF- $\beta$-induced HIF-1a levels in HFL1 (Supplementary Figures S5C-E).

\section{DISCUSSION}

Pulmonary fibrosis is a chronic, progressive lung disease with limited therapeutic options (Richeldi et al., 2017). In this study, we utilized an animal model and assessment methods for pulmonary fibrosis recommended by the American Thoracic Society (Jenkins et al., 2017). We report that repetitive $\mathrm{HBO}$ exposure attenuates bleomycin-induced pulmonary fibrosis in mice, and that $\mathrm{HBO}$ exposure, both in vivo and in vitro, inhibits fibroblast activation and ECM production. HBO therapy is generally very safe (Camporesi, 2014; Hadanny et al., 2016; Hadanny et al., 2019) and has been used in a variety of clinical practices (Choudhury, 2018; Kirby, 2019). Together with earlier reports indicating an improvement of pulmonary function in IPF patients following HBO therapy (Ma and Du, 2003; Qiu et al., 2013), our findings support HBO as a potential therapy for patients with pulmonary fibrosis.

As a master regulator of fibroblast activation, it was previously reported that in human lung fibroblasts, TGF- $\beta$ upregulates the protein levels of HIF-1 $\alpha$ and synergistically increases the expression of myofibroblast markers and ECM genes 
(Senavirathna et al., 2020). In addition, TGF- $\beta$-induced fibroblast activation is suppressed by HIF-1 $\alpha$ inhibition in human lung fibroblasts (Yamazaki et al., 2017). With evidence in this study showing the ability of $\mathrm{HBO}$ to prevent and reverse TGF$\beta$-induced HIF-1 $\alpha$ expression, we propose that HBO exposure affects TGF- $\beta$-induced fibroblast activation by modulating the expression of HIF-1a.

In addition to the effect of counteracting the upregulation of HIF- $1 \alpha$ induced by TGF- $\beta$, HBO is reported to reduce HIF- $1 \alpha$ levels through alleviating tissue hypoxia, in a similar manner to multiple ischemic conditions, injuries, and inflammatory conditions ( $\mathrm{Li}$ et al., 2005; Calvert et al., 2006; Sun et al., 2008; Bai et al., 2009; Zhou et al., 2013). Hypoxia is a hallmark of pulmonary fibrosis. Previously, studies have shown that the hypoxia signaling pathway was activated in IPF patients (Tzouvelekis et al., 2007; Ueno et al., 2011; Xie et al., 2013; Qian et al., 2015; Kusko et al., 2016; Philip et al., 2017; Yamazaki et al., 2017; Burman et al., 2018; Aquino-Galvez et al., 2019), Further, chronic exposure to hypoxic conditions can increase the severity of bleomycin-induced pulmonary fibrosis in murine models (Braun et al., 2018; Burman et al., 2018; Gille et al., 2018). Furthermore, inhibition of HIF-1a, directly or indirectly, alleviates pulmonary fibrosis in the bleomycin-induced model (Yamazaki et al., 2017; Goodwin et al., 2018; Strowitzki et al., 2019; Kseibati et al., 2020). Also, hypoxia induced fibroblast differentiation directly and this effect depended on HIF-1a (Robinson et al., 2012; Lv et al., 2018). $\mathrm{HBO}$ is an effective way of oxygenating hypoxic tissues through increasing the dissolved oxygen in plasma and amplifying oxygen diffusion distance under higher pressure. Its effect on alleviating tissue hypoxia has been confirmed in solid tumors (Kinoshita et al., 2000; Beppu et al., 2002; Thews and Vaupel, 2016) and focal cerebral ischemia tissue (Sun et al., 2008).

Given both hypoxia and TGF- $\beta$ signaling pathways are activated in pulmonary fibrosis, HBO may inhibit HIF-1 $\alpha$ expression induced by both hypoxia and TGF- $\beta$. Previous studies suggested that the effect on alleviating tissue hypoxia by $\mathrm{HBO}$ can only maintain for a certain time (Kinoshita et al., 2000; Beppu et al., 2002; Thews and Vaupel, 2016), suggesting that repetitive $\mathrm{HBO}$ exposure is required. Future studies are needed to optimize the protocol for the clinical application of applying $\mathrm{HBO}$ as a therapy for pulmonary fibrosis.

In summary, this study provides evidence that $\mathrm{HBO}$ exposure attenuates bleomycin-induced pulmonary fibrosis in vivo and TGF- $\beta$-induced fibroblast activation in vitro. Mechanistically, this effect may be mediated by downregulating TGF- $\beta$-induced expression of HIF-1 $\alpha$. These findings support $\mathrm{HBO}$ as a potential

\section{REFERENCES}

Aquino-Gálvez, A., González-Ávila, G., Jiménez-Sánchez, L. L., Maldonado-Martínez, H. A., Cisneros, J., Toscano-Marquez, F., et al. (2019). Dysregulated Expression of Hypoxia-Inducible Factors Augments Myofibroblasts Differentiation in Idiopathic Pulmonary Fibrosis. Respir. Res. 20 (1), 130. doi:10.1186/s12931-019-1100-4

Bai, X., Sun, B., Pan, S., Jiang, H., Wang, F., Krissansen, G. W., et al. (2009). DownRegulation of Hypoxia-Inducible Factor-1a by Hyperbaric Oxygen Attenuates life-changing therapy for patients with pulmonary fibrosis, although further research is needed to fully evaluate this.

\section{DATA AVAILABILITY STATEMENT}

Publicly available datasets were analyzed in this study. These data can be found in the Gene Expression Omnibus (GEO) database: accession code GSE143348.

\section{ETHICS STATEMENT}

The animal study was reviewed and approved by the Animal Ethics Committee at Nantong University.

\section{AUTHOR CONTRIBUTIONS}

YW, ZJ, YY, and YL conceived and designed the experiments. YY, YL, GQ, YZ, and ZX conducted the experiments. YY and YZ analyzed the data. YY, YW, YL, and $\mathrm{CH}$ wrote the manuscript. All authors read and approved the manuscript.

\section{FUNDING}

YY was supported by the Natural Science Fund for Colleges and Universities in Jiangsu Province (19KJB320002), the Science and Technology Planning Project of Nantong Municipality, China (JC2020010), and a Research Startup Fund of Nantong University. YZ was supported by an Institute for Life Sciences PhD Studentship. ZX was supported by the China Scholarship Council. $\mathrm{CH}$ was supported by the Gerald Kerkut Charitable Trust and University of Southampton Central VC Scholarship Scheme. ZJ was supported by the National Natural Science Foundation of China (81671859 and 81601639) and the Natural Science Foundation of Jiangsu Province (BK20161282). YW was supported by the Medical Research Council (MR/S025480/1).

\section{SUPPLEMENTARY MATERIAL}

The Supplementary Material for this article can be found online at: https://www.frontiersin.org/articles/10.3389/fmolb.2021.675437/ full\#supplementary-material

the Severity of Acute Pancreatitis in Rats. Pancreas 38 (5), 515-522. doi:10. 1097/MPA.0b013e31819cac24

Bell, E. C., Cox, N. S., Goh, N., Glaspole, I., Westall, G. P., Watson, A., et al. (2017). Oxygen Therapy for Interstitial Lung Disease: a Systematic Review. Eur. Respir. Rev. 26 (143), 160080. doi:10.1183/16000617.00802016

Beppu, T., Kamada, K., Yoshida, Y., Arai, H., Ogasawara, K., and Ogawa, A. (2002). Change of Oxygen Pressure in Glioblastoma Tissue under Various Conditions. J. Neurooncol. 58 (1), 47-52. doi:10.1023/a:1015832726054 
Braun, R. K., Broytman, O., Braun, F. M., Brinkman, J. A., Clithero, A., Modi, D., et al. (2018). Chronic Intermittent Hypoxia Worsens Bleomycin-Induced Lung Fibrosis in Rats. Respir. Physiol. Neurobiol. 256, 97-108. doi:10.1016/j.resp. 2017.04.010

Burman, A., Kropski, J. A., Calvi, C. L., Serezani, A. P., Pascoalino, B. D., Han, W., et al. (2018). Localized Hypoxia Links ER Stress to Lung Fibrosis through Induction of C/EBP Homologous Protein. JCI Insight 3 (16), e99543. doi:10. 1172/jci.insight. 99543

Calvert, J. W., Cahill, J., Yamaguchi-Okada, M., and Zhang, J. H. (2006). Oxygen Treatment after Experimental Hypoxia-Ischemia in Neonatal Rats Alters the Expression of HIF-1 $\alpha$ and its Downstream Target Genes. J. Appl. Physiol. 101 (3), 853-865. doi:10.1152/japplphysiol.00268.2006

Camporesi, E. M. (2014). Side Effects of Hyperbaric Oxygen Therapy. Undersea Hyperb. Med. 41 (3), 253-257.

Choudhury, R. (2018). Hypoxia and Hyperbaric Oxygen Therapy: a Review. Ijgm 11, 431-442. doi:10.2147/IJGM.S172460

Dowman, L. M., McDonald, C. F., Bozinovski, S., Vlahos, R., Gillies, R., Pouniotis, D., et al. (2017). Greater Endurance Capacity and Improved Dyspnoea with Acute Oxygen Supplementation in Idiopathic Pulmonary Fibrosis Patients without Resting Hypoxaemia. Respirology 22 (5), 957-964. doi:10.1111/resp. 13002

Faverio, P., De Giacomi, F., Bonaiti, G., Stainer, A., Sardella, L., Pellegrino, G., et al. (2019). Management of Chronic Respiratory Failure in Interstitial Lung Diseases: Overview and Clinical Insights. Int. J. Med. Sci. 16 (7), 967-980. doi:10.7150/ijms.32752

George, P. M., Wells, A. U., and Jenkins, R. G. (2020). Pulmonary Fibrosis and COVID-19: the Potential Role for Antifibrotic Therapy. Lancet Respir. Med. 8 (8), 807-815. doi:10.1016/S2213-2600(20)30225-3

Gille, T., Didier, M., Rotenberg, C., Delbrel, E., Marchant, D., Sutton, A., et al. (2018). Intermittent Hypoxia Increases the Severity of Bleomycin-Induced Lung Injury in Mice. Oxidative Med. Cell Longevity 2018, 1-13. doi:10.1155/ 2018/1240192

Goodwin, A. T., and Jenkins, G. (2016). Molecular Endotyping of Pulmonary Fibrosis. Chest 149 (1), 228-237. doi:10.1378/chest.15-1511

Goodwin, J., Choi, H., Hsieh, M.-h., Neugent, M. L., Ahn, J.-M., Hayenga, H. N., et al. (2018). Targeting Hypoxia-Inducible Factor-1 $\alpha /$ Pyruvate Dehydrogenase Kinase 1 Axis by Dichloroacetate Suppresses Bleomycin-Induced Pulmonary Fibrosis. Am. J. Respir. Cel Mol Biol 58 (2), 216-231. doi:10.1165/rcmb.2016-0186OC

Hadanny, A., Meir, O., Bechor, Y., Fishlev, G., Bergan, J., and Efrati, S. (2016). The Safety of Hyperbaric Oxygen Treatment-Rretrospective Analysis in 2,334 Patients. Undersea Hyperb. Med. 43 (2), 113-122.

Hadanny, A., Zubari, T., Tamir-Adler, L., Bechor, Y., Fishlev, G., Lang, E., et al. (2019). Hyperbaric Oxygen Therapy Effects on Pulmonary Functions: a Prospective Cohort Study. BMC Pulm. Med. 19 (1), 148. doi:10.1186/ s12890-019-0893-8

Hänzelmann, S., Castelo, R., and Guinney, J. (2013). GSVA: Gene Set Variation Analysis for Microarray and RNA-Seq Data. BMC Bioinformatics 14, 7. doi:10. 1186/1471-2105-14-7

Hübner, R.-H., Gitter, W., Eddine El Mokhtari, N., Mathiak, M., Both, M., Bolte, H., et al. (2008). Standardized Quantification of Pulmonary Fibrosis in Histological Samples. Biotechniques 44 (4), 507-514. doi:10.2144/ 000112729

Jenkins, R. G., Moore, B. B., Chambers, R. C., Eickelberg, O., Königshoff, M., Kolb, M., et al. (2017). An Official American Thoracic Society Workshop Report: Use of Animal Models for the Preclinical Assessment of Potential Therapies for Pulmonary Fibrosis. Am. J. Respir. Cel Mol Biol 56 (5), 667-679. doi:10.1165/ rcmb.2017-0096ST

Kinoshita, Y., Kohshi, K., Kunugita, N., Tosaki, T., and Yokota, A. (2000). Preservation of Tumour Oxygen after Hyperbaric Oxygenation Monitored by Magnetic Resonance Imaging. Br. J. Cancer 82 (1), 88-92. doi:10.1054/bjoc. 1999.0882

Kirby, J. P. (2019). Hyperbaric Oxygen Therapy as an Elective Treatment. Mo. Med. 116 (3), 184-187.

Koyauchi, T., Hasegawa, H., Kanata, K., Kakutani, T., Amano, Y., Ozawa, Y., et al. (2018). Efficacy and Tolerability of High-Flow Nasal Cannula Oxygen Therapy for Hypoxemic Respiratory Failure in Patients with Interstitial Lung Disease with Do-Not-Intubate Orders: A Retrospective Single-Center Study. Respiration 96 (4), 323-329. doi:10.1159/000489890
Kseibati, M. O., Shehatou, G. S. G., Sharawy, M. H., Eladl, A. E., and Salem, H. A. (2020). Nicorandil Ameliorates Bleomycin-Induced Pulmonary Fibrosis in Rats through Modulating eNOS, iNOS, TXNIP and HIF-1a Levels. Life Sci. 246, 117423. doi:10.1016/j.lfs.2020.117423

Kusko, R. L., Brothers, J. F., 2nd, Tedrow, J., Pandit, K., Huleihel, L., Perdomo, C., et al. (2016). Integrated Genomics Reveals Convergent Transcriptomic Networks Underlying Chronic Obstructive Pulmonary Disease and Idiopathic Pulmonary Fibrosis. Am. J. Respir. Crit. Care Med. 194 (8), 948-960. doi:10.1164/rccm.201510-2026OC

Lam, G., Fontaine, R., Ross, F. L., and Chiu, E. S. (2017). Hyperbaric Oxygen Therapy: Exploring the Clinical Evidence. Adv. Skin Wound Care 30 (4), 181-190. doi:10.1097/01.ASW.0000513089.75457.22

Li, Y., Zhou, C., Calvert, J. W., Colohan, A. R. T., and Zhang, J. H. (2005). Multiple Effects of Hyperbaric Oxygen on the Expression of HIF-1a and Apoptotic Genes in a Global Ischemia-Hypotension Rat Model. Exp. Neurol. 191 (1), 198-210. doi:10.1016/j.expneurol.2004.08.036

Lv, X.-m., Li, M.-d., Cheng, S., Liu, B.-l., Liu, K., Zhang, C.-f., et al. (2018). Neotuberostemonine Inhibits the Differentiation of Lung Fibroblasts into Myofibroblasts in Mice by Regulating HIF-1a Signaling. Acta Pharmacol. Sin 39 (9), 1501-1512. doi:10.1038/aps.2017.202

$\mathrm{Ma}, \mathrm{Y}$., and Du, J. (2003). Hyperbaric Oxygen Treatment on Idiopathic Pulmonary Fibrosis: Clinical Observation of 67 Cases. Shandong Med. J. 43 (1), 34

Meltzer, E. B., and Noble, P. W. (2008). Idiopathic Pulmonary Fibrosis. Orphanet J. Rare Dis. 3, 8. doi:10.1186/1750-1172-3-8

Oscarsson, N., Ny, L., Mölne, J., Lind, F., Ricksten, S.-E., Seeman-Lodding, H., et al. (2017). Hyperbaric Oxygen Treatment Reverses Radiation Induced Pro-fibrotic and Oxidative Stress Responses in a Rat Model. Free Radic. Biol. Med. 103, 248-255. doi:10.1016/j.freeradbiomed.2016.12.036

Philip, K., Mills, W. T., Davies, J., Chen, N. Y., Karmouty-Quintana, H., Luo, F., et al. (2017). HIF1A Up-regulates the ADORA2B Receptor on Alternatively Activated Macrophages and Contributes to Pulmonary Fibrosis. FASEB j. 31 (11), 4745-4758. doi:10.1096/fj.201700219R

Qian, F., He, M., Duan, W., Mao, L., Li, Q., Yu, Z., et al. (2015). Cross Regulation between Hypoxia-Inducible Transcription Factor-1 $\alpha$ (HIF-1 $\alpha$ ) and Transforming Growth Factor (TGF)-B1 Mediates Nickel Oxide Nanoparticles (NiONPs)Induced Pulmonary Fibrosis. Am. J. Transl Res. 7 (11), 2364-2378.

Qiu, X., Huang, H., and Jin, N. (2013). Obervation of Effect of Hyperbaric Oxygen Therapy on Serum Fibrosis Associated Indexes and Pulmonary Function in Idiopathic Pulmonary Fibrosis Patients. J. Hainan Med. Univ. 19 (8), 1054

Richeldi, L., Collard, H. R., and Jones, M. G. (2017). Idiopathic Pulmonary Fibrosis. The Lancet 389 (10082), 1941-1952. doi:10.1016/S0140-6736(17)30866-8

Robinson, C. M., Neary, R., Levendale, A., Watson, C. J., and Baugh, J. A. (2012). Hypoxia-induced DNA Hypermethylation in Human Pulmonary Fibroblasts Is Associated with Thy-1 Promoter Methylation and the Development of a Profibrotic Phenotype. Respir. Res. 13, 74. doi:10.1186/1465-9921-13-74

Senavirathna, L. K., Huang, C., Pushparaj, S., Xu, D., and Liu, L. (2020). Hypoxia and Transforming Growth Factor $\beta 1$ Regulation of Long Non-coding RNA Transcriptomes in Human Pulmonary Fibroblasts. Physiol. Rep. 8 (1), e14343. doi:10.14814/phy2.14343

Strowitzki, M. J., Ritter, A. S., Kimmer, G., and Schneider, M. (2019). Hypoxiaadaptive Pathways: A Pharmacological Target in Fibrotic Disease?. Pharmacol. Res. 147, 104364. doi:10.1016/j.phrs.2019.104364

Sun, L., Marti, H. H., and Veltkamp, R. (2008). Hyperbaric Oxygen Reduces Tissue Hypoxia and Hypoxia-Inducible Factor-1a Expression in Focal Cerebral Ischemia. Stroke 39 (3), 1000-1006. doi:10.1161/STROKEAHA.107.490599

Thews, O., and Vaupel, P. (2016). Temporal Changes in Tumor Oxygenation and Perfusion upon Normo- and Hyperbaric Inspiratory Hyperoxia. Strahlenther Onkol 192 (3), 174-181. doi:10.1007/s00066-015-0916-1

Tzouvelekis, A., Harokopos, V., Paparountas, T., Oikonomou, N., Chatziioannou, A., Vilaras, G., et al. (2007). Comparative Expression Profiling in Pulmonary Fibrosis Suggests a Role of Hypoxia-Inducible Factor-1 $\alpha$ in Disease Pathogenesis. Am. J. Respir. Crit. Care Med. 176 (11), 1108-1119. doi:10.1164/rccm.200705-683OC

Ueno, M., Maeno, T., Nomura, M., Aoyagi-Ikeda, K., Matsui, H., Hara, K., et al. (2011). Hypoxia-inducible Factor-1 $\alpha$ Mediates TGF- $\beta$-Induced PAI-1 Production in Alveolar Macrophages in Pulmonary Fibrosis. Am. J. Physiology-Lung Cell Mol. Physiol. 300 (5), L740-L752. doi:10.1152/ajplung.00146.2010

Vandivort, T. C., An, D., and Parks, W. C. (2016). An Improved Method for Rapid Intubation of the Trachea in Mice. JoVE 108, 53771. doi:10.3791/53771 
Visca, D., Mori, L., Tsipouri, V., Fleming, S., Firouzi, A., Bonini, M., et al. (2018). Effect of Ambulatory Oxygen on Quality of Life for Patients with Fibrotic Lung Disease (AmbOx): a Prospective, Open-Label, Mixed-Method, Crossover Randomised Controlled Trial. Lancet Respir. Med. 6 (10), 759-770. doi:10. 1016/S2213-2600(18)30289-3

Xie, H., Tan, J.-t., Wang, R.-l., Meng, X.-X., Tang, X., and Gao, S. (2013). Expression and Significance of HIF-1 $\alpha$ in Pulmonary Fibrosis Induced by Paraquat. Exp. Biol. Med. (Maywood) 238 (9), 1062-1068. doi:10.1177/ 1535370213498978

Yamazaki, R., Kasuya, Y., Fujita, T., Umezawa, H., Yanagihara, M., Nakamura, H., et al. (2017). Antifibrotic Effects of Cyclosporine A on TGF- $\beta 1$-Treated Lung Fibroblasts and Lungs from Bleomycin-treated Mice: Role of Hypoxiainducible Factor-1a. FASEB j. 31 (8), 3359-3371. doi:10.1096/fj.201601357R

Yuan, Y., Zhou, Y., Li, Y., Hill, C., Ewing, R. M., Jones, M. G., et al. (2020). Deconvolution of RNA-Seq Analysis of Hyperbaric Oxygen-Treated Mice Lungs Reveals Mesenchymal Cell Subtype Changes. Ijms 21 (4), 1371. doi:10.3390/ijms21041371

Zhou, Y., Liu, X. H., Qu, S. D., Yang, J., Wang, Z. W., Gao, C. J., et al. (2013). Hyperbaric Oxygen Intervention on Expression of Hypoxia-Inducible Factor-
$1 \alpha$ and Vascular Endothelial Growth Factor in Spinal Cord Injury Models in Rats. Chin. Med. J. (Engl) 126 (20), 3897-3903. doi:10.3760/cma.j.issn.03666999.20130571

Zou, R. H., Kass, D. J., Gibson, K. F., and Lindell, K. O. (2020). The Role of Palliative Care in Reducing Symptoms and Improving Quality of Life for Patients with Idiopathic Pulmonary Fibrosis: A Review. Pulm. Ther. 6 (1), 35-46. doi:10.1007/s41030-019-00108-2

Conflict of Interest: The authors declare that the research was conducted in the absence of any commercial or financial relationships that could be construed as a potential conflict of interest.

Copyright (C) 2021 Yuan, Li, Qiao, Zhou, Xu, Hill, Jiang and Wang. This is an openaccess article distributed under the terms of the Creative Commons Attribution License (CC BY). The use, distribution or reproduction in other forums is permitted, provided the original author(s) and the copyright owner(s) are credited and that the original publication in this journal is cited, in accordance with accepted academic practice. No use, distribution or reproduction is permitted which does not comply with these terms. 